\title{
Adjustment of Transfer Tools for the Production of Micro- and Macroarrays
}

BioTechniques 31:848-854 (October 2001)

\author{
Aleksey V. Soldatov ${ }^{1}$, Elena \\ N. Nabirochkina ${ }^{1,2}$, Sofia G. \\ Georgieva $^{1,2}$, and \\ Holger Eickhoff ${ }^{3}$ \\ ${ }^{1}$ Max-Planck-Institut für \\ Molekulare Genetik, Berlin, \\ ${ }^{2}$ University of Oslo, Center for \\ Medical Studies, Moscow, \\ Russia, and ${ }^{3}$ Scienion AG, \\ Berlin, Germany
}

\author{
ABSTRACT \\ A transfer tool adjustment procedure for \\ the generation of micro- and macroarrays is \\ described. It is based on control spotting of \\ solutions containing radioactive or fluores- \\ cent labels and the quantification of each \\ obtained spot by standard image-analyzing \\ software. This method provides a simple, \\ rapid, and efficient way to control the quali- \\ ty and liquid delivery properties of spotting \\ transfer tools.
}

\section{INTRODUCTION}

Array-based expression profiling is a widely accepted and powerful tool in modern molecular biology $(3,5,7)$. For the production of macro- and microarrays, standard Cartesian robotic systems transfer DNA samples stored in microplates onto planar surfaces such as nylon membranes or modified glass slides by the use of spotting tools (2). These tools consist of a holder that carries up to 384 pins. These pins can be either solid or split and are manufactured from a broad range of materials, including stainless steel, tungsten, and silicon. Before transfer, the pins are loaded in the microplates by simply dipping the pins into the DNA or protein solutions. The adherent liquid at the print tip end is subsequently transferred through printing it onto the planar surfaces. When compared to split pins, solid print tips are cheaper and much easier to clean and were therefore used in this study. The availability and the competitive pricing of solid spotting pins especially has made spotting procedures more common in biological laboratories when compared to other arraying procedures such as inkjet printing.

The volume that is held by the tip of a pin is proportional to the cube of the diameter of the tip. As a result, small differences in print tip diameters lead to severe differences in the transferred volumes. This is especially important for small diameters that are required for the production of high-density macro- and microarrays. Thus, the individual control of spotting pins in robotic transfer tools is required. With current protocols, this control is very time and labor intensive for 384-pin heads that are typically used for the preparation of macroarrays.

Instead of using methods such as individual pin tip control under a microscope or spotting of ink solutions, we suggest a precise and fast procedure that utilizes only standard facilities present in an expression-profiling laboratory.

\section{MATERIALS AND METHODS}

As starting material, approximately 1.5 times the number of individual pins that are loaded into one spotting gadget should be used. Spotting (QBot; Genetix, New Milton, UK) was performed on nylon Hybond ${ }^{\circledR}-\mathrm{N}^{+}$membranes (Amersham Pharmacia Biotech, Little Chalfont, UK) with a 384-pin gridding head (X5251 - $0.25 \mathrm{~mm}$; Genetix). For liquid transfer in the test system, a solution containing radioactively labeled dATP was used for membrane spotting $(1 \times$ PCR buffer containing $0.5 \mathrm{M} \mathrm{NaOH}$ and $2 \mu \mathrm{Ci} / \mathrm{mL}$ $\alpha\left[{ }^{32} \mathrm{P}\right]-d A T P$; the $2.5 \times \mathrm{PCR}$ buffer consisted of $125 \mathrm{mM}$ Tris-HCl, $\mathrm{pH} 8.6,125$ $\mathrm{mM} \mathrm{KCl}, 3.75 \mathrm{mM} \mathrm{MgCl}_{2}, 0.25 \%$ Tween ${ }^{\circledR}$ 20, $3.75 \mathrm{M}$ betaine, $125 \mu \mathrm{M}$ Cresol red. Any other [32P]- or [33P]-labeled compounds may be used instead of $\alpha\left[{ }^{32} \mathrm{P}\right]-d A T P$. Transfer was performed 10 times on the same position, which is identical to the typical protocol 
for spotting with blunt-end pins. After spotting, the membranes were exposed to Fuji Imaging Plates ${ }^{\mathrm{TM}}$, scanned with the BAS-5000 ${ }^{\mathrm{TM}}$ Bio-Imaging analyzer and quantified by the AIDA ${ }^{\mathrm{TM}}$ develop- ment software (Raytest Isotopenmessgeräte $\mathrm{GmbH}$, Straubenhardt, Germany). Data was imported into $\mathrm{Mi}$ crosoft $^{\circledR}$ Excel $^{\circledR} 2000$ spreadsheets for further statistical analysis.

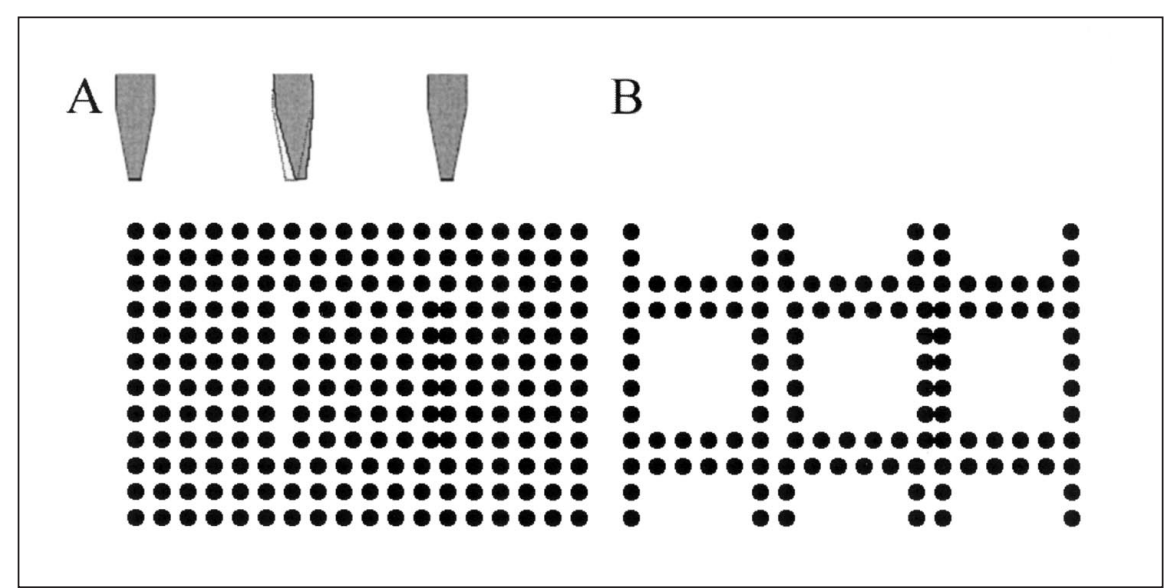

Figure 1. Scheme of control of pins bending. (A) Bent pins will induce a shift of the corresponding block of dots during spotting. (B) Fragment of the picture that will be obtained after control ink spotting of perimeter dots only.

\section{RESULTS}

Our aim was to select 384 pins with nearly identical liquid transfer properties, to assemble one 384-pin transfer tool (gadget). We routinely use this gadget for the preparation of nylonbased microarrays of PCR-amplified cDNA fragments with the density of about 120 elements/ $\mathrm{cm}^{2}(4,6)$.

The procedure for the transfer tool adjustment includes ( $i$ ) test spotting with all available pins of a given diameter, (ii) quantification of the dots and assessment of the transfer volume to each pin, (iii) selection of the set with the minimal volume variation, and (iv) assembling the pin head and quality control via a test spotting.

\section{Test Spotting}

Spotting was performed in conditions that simulated a real macroarray 
fabrication procedure. To prepare an adjusted pin head, it is necessary to have more than one complete set of the pins, preferably of more than $1.5 \times$ excess. In the first alignment round, spotting is performed with one full set (384) of pins in the transfer tool. These pins are then substituted by the next set of pins, and the transfer is repeated. If the number of additional pins is not sufficient for a full second set, then the new pins are substituted for some of the previously tested pins.

After this test run, the tested pins were accurately placed in 384-well plates, to maintain their coordinates in the test runs.

There is one more parameter of the pins that should be controlled. Higher densities may be achieved when the spots are equidistant. The even distribution is disturbed when the tip of some pin is slightly bent because this induces a shift of the corresponding block (Figure $1 \mathrm{~A})$. Bended pins may be identified directly in test spotting or with additional control ink spotting. For a quick quality check, it is sufficient to spot just a perimeter of a spotting block (Figure 1B) because the distribution of the spots within the blocks is the same for the whole macroarray and depends only on the precision of the robot's movements. Gaps between blocks are examined, and bended pins are excluded from further analysis.

\section{Quantification}

Software that is normally used for the quantification of expression profiles was used for the quantification of the signals on test membranes. It is also possible to perform not only relative but also absolute measurement of the transferred volume if putting the known volume of the labeled solution on the same membrane and determining the radioactive signal from the obtained spot. To keep within the linear range of the BAS-5000 Bio-Imaging analyzer, we prefer to use dilution series instead of one spot (see Figure 2 for results).

\section{Selection of Pins}

Before the final pin selection, the quantified spotting data are organized in an Excel spreadsheet in two columns.
The first column displays the pin number, including its coordinates in the transfer tool. The transferred volumes, meaning the intensity of the signal on the test membrane, are stored in the second column. The result table is then sorted according to the transferred volume, which is displayed in Figure 3. The next step is to find a 384-row interval, for which the deviation of transferred liquid volumes is minimal. We calculated these deviations for all possible intervals as the ratio of the transferred volume of the first pin in the interval (column " $x$ " in Figure 3) to that of the last pin (row " $x+383$ " in Figure 3 ). The minimal value of the calculated ratio corresponds to the optimal set of spotting pins.

\section{Assembling the Pin Head}

The pin head is finally assembled from the pins of the chosen interval (Figure 3). Additionally, about 10 pins from both sides of interval are taken as a reserve. This enables one to repair the transfer gadget quickly in case of damage to individual pins, without requiring a new adjustment.

\section{DISCUSSION}

The volumes transferred by the pins depend not only on the pin diameter but also on parameters such as liquid viscosity and surface tension. For the mixture mentioned in the Materials and Methods section, the volume transferred in one print cycle well fits to the formula: $\mathrm{V}[\mathrm{nL}]=186 \times(\mathrm{d}[\mathrm{mm}])^{3}$ (experimental data: $2.2,3.2$, and $13 \mathrm{~nL}$ for 0.23 -, $0.25-$, and $0.42-\mathrm{mm}$ pins, respectively).

It is interesting to estimate the surface density of the probe (DNA molecules) that will be obtained for different pin diameters. The probe density is proportional to the transferred volume (V) and inversely proportional to the area (S) of the spot. The size of the spot increases with the diameter of the pin. Thus, the probe density will be proportional to $\mathrm{V} / \mathrm{S} \sim \mathrm{d}^{3} / \mathrm{d}^{(<) 2} \sim \mathrm{d}^{(>) 1}$. Thus, to obtain the same surface density of the probe on the carrier, it is necessary to perform more transfers with the small pins than with the large ones. In practice, the densities produced by pins of standard diameters of $0.15,0.25,0.4 \mathrm{~mm}$ relate to $1: 2: 4$.

At present, the use of membranes

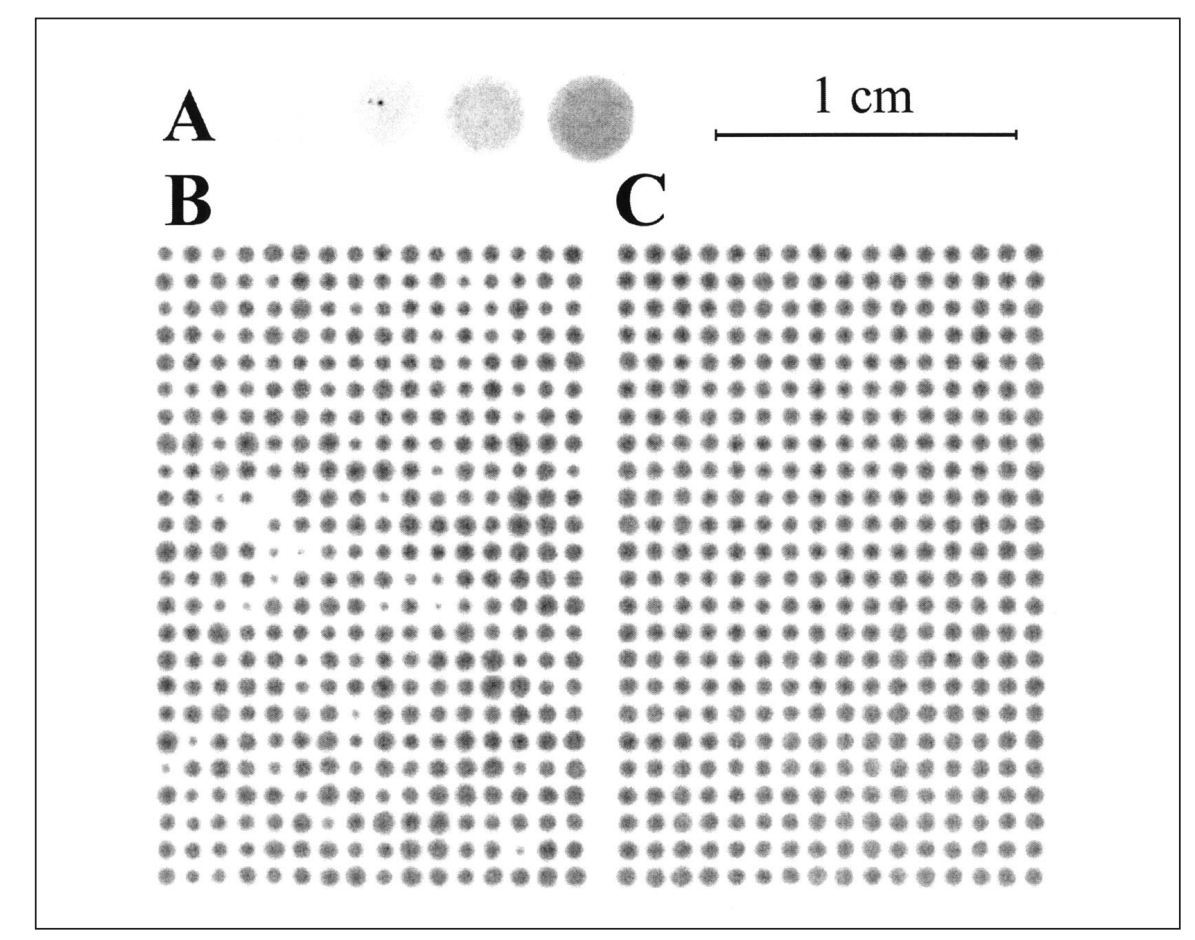

Figure 2. Dots obtained with gadget before and after adjustment. (A) Two times dilution series of the labeled solution used for the absolute quantification of the transferred volume. Solution $(0.5 \mu \mathrm{L})$ was loaded in each spot. (B and C) The dots obtained with gadget before and after adjustment. Dots are the pictures of individual spots detected with the BAS-5000 Bio-Imaging analyzer. On the original membrane, they were $4.5 \mathrm{~mm}$ apart. On the picture, they were placed close together for presentation. 
for the construction of microarrays is not as popular as the use of glass. The main reason is the low autofluorescence of glass that makes possible work with a fluorescent label, in particular to hybridize one microarray simultaneously with two samples labeled with different fluorescent dyes (8). Another advantage of glass supports is their rigidity and hardness, which enables researchers to prepare microarrays with high density. However, membranes have some clear advantages. First, preparing DNA for the spotting process is much easier (no purification of PCR products, just adding of $\mathrm{NaOH}$ to the PCR mixture), and the binding capacity of membranes is significantly higher when compared to any glass surface (1). Second, the price of membranes is much lower than the price of glass with a special coating for covalent DNA binding. Thus, in the case of the small series of microarrays with the large number of elements, membranes are doubtlessly more convenient than glass.

Signals on a membrane are directly proportional to the transferred volume. Figure 3 shows the distribution of signals for 7680.25 -mm pins. For the selected 384 pins with minimal volume variation, the ratio of the highest signal to the lowest is 1:2. A further increase in the number of tested pins will result in an even narrower distribution.

The method described here uses spotting on nylon membranes and radioactive detection. The procedure controls the pin quality in conditions identical to thsoe used for a real microarray experiment. The process can be easily adapted to any other surfaces and to the detection methods used in the particular laboratory. Consequently, the adjustment procedure may also be used for other microarray-generating instruments (e.g., split pins and capillary transfer).

The method described here might be important for pin manufacturers. Testing a large number of pins using this method will result in subsets of nearly equivalent pins. Although these subsets will differ in diameter, this does not matter for the practical use as long as they are offered according to their real size.

\section{ACKNOWLEDGMENTS}

We thank E. Rohlfs for the assistance in robot handling and N. Tandon for helpful discussions. The work of E.N. and S.G. was supported by the fellowship from Oslo University and grant no. N 00-04-48066 from the Russian Foundation for Basic Research; A.S., EMBO fellowship ALTF517-1999; A.S. and H.E., BMBF grant no. 0312275F/2.

\section{REFERENCES}

1.Baldwin, D., V. Crane, and D. Rice. 1999. A comparison of gel-based, nylon filter and microarray techniques to detect differential RNA expression in plants. Curr. Opin. Plant Biol. 2:96-103

2.Bowtell, D.D. 1999. Options available-from start to finish-for obtaining expression data by microarray. Nat. Genet. 21(1 Suppl):25-32.

3.Duggan, D.J., M. Bittner, Y. Chen, P. Meltzer, and J.M. Trent. 1999. Expression profiling using cDNA microarrays. Nat. Genet. 21(1 Suppl):10-14.

4.Eickhoff, H., J. Schuchhardt, I. Ivanov, S. Meier-Ewert, J. O'Brien, A. Malik, N. Tandon, E.W. Wolski et al. 2000. Tissue gene expression analysis using arrayed normalized cDNA libraries. Genome Res. 10:1230-1240.

5.Freeman, W.M., D.J. Robertson, and K.E. Vrana. 2000. Fundamentals of DNA hybridization arrays for gene expression analysis. BioTechniques 29:1042-1055.

6.Schuchhardt, J., D. Beule, A. Malik, E. Wolski, H. Eickhoff, H. Lehrach, and $H$. Herzel. 2000. Normalization strategies for cDNA microarrays. Nucleic Acids Res. 28:E47.

7.van Berkum, N.L. and F.C. Holstege. 2001. DNA microarrays: raising the profile. Curr. Opin. Biotechnol. 12:48-52.

8.Winzeler, E.A., M. Schena, and R.W. Davis. 1999. Fluorescence-based expression monitoring using microarrays. Methods Enzymol. 306:3-18.

Received 2 March 2001; accepted 25 June 2001.

Address correspondence to:

Dr. Holger Eickhoff

Scienion AG

Volmerstr. $7 a$

12989 Berlin, Germany

e-mail: eickhoff@scienion.com

Figure 3. Signal intensities for different pins. Signals are distributed continuously from 0 to 735 . Selected pins are marked. Signals in this interval are distributed from 441 to 524 .

For reprints of this or any other article, contact Reprints@BioTechniques.com 